\title{
Using First Passage Times to Manage Eco-system Regime Shifts
}

\author{
Tua Tamba and M.D. Lemmon
}

\begin{abstract}
Regime shifts refer to sudden changes in the structure or function of an eco-system due to external forces on the system. Such shifts arise because these systems have multiple equilibria so external disturbances may drive the state between different regions of attraction. Examples of such shifts include the shift in eutrophic state of shallow lakes in response to nutrient loading as well as the collapse of fisheries in response to the introduction of invasive species. A key measure of an ecosystem's resilience to such shifts is measured by first passage times (FPT) between the basins of attraction for different equilibria. Prior work in eco-system management has assumed low-dimensional linearized models driven by Brownian motions. This paper uses sum-of-square (SOS) programs to bound FPT probabilities for more complex nonlinear population processes in which the primary disturbance is a Poisson jump process. The paper uses this approach to design management policies controlling the level of invasive species in lake systems.
\end{abstract}

\section{INTRODUCTION}

Regime shifts [13] refer to the abrupt change in an ecosystem's state as a result of external disturbances. Such shifts arise when the underlying system has multiple equilibria and when disturbances drive the system state between the basins of attraction for competing equilibria. These conditions are nearly always present in an eco-system's population processes. In many cases, regime shifts occur with great speed as a result of slow changes in the environmental conditions. A prime example of this is seen in the eutrophication of shallow lakes caused by nutrient loading [14] where a previously clear lake with a high level of biodiversity abruptly shifts to a turbid state whose biota is dominated by algae. Another example can be found when invasive species invade an existing eco-system, thereby causing abrupt declines in those species that have historically occupied the system [12]. Each of these shifts has the potential to greatly disrupt the services that these eco-systems provide to society. There is, therefore, considerable interest in finding ways to detect such shifts and to develop policies to better manage the impact these shifts have on eco-system services [4].

Eco-system resilience may be measured by those disturbance intensities that trigger a shift to an alternative equilibrium [6]. It may also be measured by the time it takes a system to return to normal after a given disturbance [4]. For either definition, one must realize that disturbances are usually not one-time events. Eco-system disturbances are better characterized as stochastic renewal processes that deliver a sequence of "shocks" to the system. A good example

The authors are with Department of Electrical Engineering, University of Notre Dame, IN 46556, USA; e-mails: (ttamba,lemmon)@nd.edu. The authors gratefully acknowledge the partial financial support of Notre Dame's Environmental Change Initiative and the National Science Foundation (CNS-1239222) of this is found in random storm events that deliver pulses of nutrient rich water to downstream receiving waters. For such stochastic disturbances, one should use a probabilistic measure of resilience.

One appropriate measure of resilience is given by a system's first passage times. These passage (a.k.a. exit or stopping) times are random variables representing the first time when the system state first enters the region of attraction for a competing equilibrium. This paper presents computational methods for characterizing first passage times of regime shifts in ecological systems. The main result uses sum-of-square (SOS) programming [10] to compute first passage times for jump dynamical systems. SOS programs have already been proposed as a tool for analyzing stochastic reachability problems in cellular biology [3]. This work extends the earlier methods in [9] to eco-systems modeled as jump dynamical processes. We then demonstrate how these results can be used to better manage invasive species in a specific ecological system [2].

The remainder of this paper is organized as follows. Jump processes and first passage times are reviewed in sections II and III, respectively. Section IV presents the use of sumof-square programming to characterize first passage times of jump processes. Section V illustrates how these tools may be used to manage invasive species in lake eco-systems.

Notational Conventions: Let $\mathbb{Z}$ and $\mathbb{R}$ denote the set of integers and real numbers, respectively. Let $\mathbb{Z}_{+}$and $\mathbb{R}_{+}$ denote the set of positive integers and non-negative real numbers, respectively. Let $\mathbb{R}^{n}$ denote the $n$-dimensional Euclidean vector space. Given a vector $x \in \mathbb{R}^{n}$, we let $x_{i}$ denote the $i$ th component of that vector. A continuous-time random process, $x$, will be denoted as $\left\{x(t): t \in \mathbb{R}_{+}\right\}$. The probability and expectation of this process will be denoted as $\mathbb{P}[x]$ and $\mathbb{E}[x]$, respectively. If $\{x(t)\}$ has distribution $F(x)$, then its $n$th moment is $\mathbb{M}^{n}=\int_{0}^{\infty} x^{n} d F(x)$.

An $n$-dimensional multi-index is an $n$-tuple, $\alpha \equiv$ $\left(\alpha_{1}, \alpha_{2}, \cdots, \alpha_{n}\right)$ of non-negative integers. The absolute value of a multi-index $\alpha$ is defined as $|\alpha|=\sum_{i=1}^{n} \alpha_{i}$. Given a vector $x \in \mathbb{R}^{n}$ with components $x_{i}(i=1,2, \ldots, n)$ and an $n$-dimensional multi-index $\alpha$, the $\alpha$ th power of $x$ is defined as $x^{[\alpha]} \equiv x_{1}^{\alpha_{1}} x_{2}^{\alpha^{2}} \cdots x_{n}^{\alpha_{n}}$. Let $\alpha ! \equiv \alpha_{1} ! \alpha_{2} ! \cdots \alpha_{n}$ !. The sum or difference of two multi-indices in $\mathbb{Z}_{+}^{n}$ is the component wise sum/difference of the indices. In a similar way, we say that $\alpha \geq \beta$ if and only if $\alpha_{i} \geq \beta_{i}$ for $i=1,2, \ldots, n$. The binomial coefficient of $\alpha$ and $\beta$ is defined as

$$
\left(\begin{array}{l}
\alpha \\
\beta
\end{array}\right)=\left(\begin{array}{l}
\alpha_{1} \\
\beta_{1}
\end{array}\right) \cdots\left(\begin{array}{l}
\alpha_{n} \\
\beta_{n}
\end{array}\right)=\frac{\alpha !}{\beta !(\alpha-\beta) !}
$$

Let $V(\cdot): \mathbb{R}^{n} \rightarrow \mathbb{R}$ be a real valued function over $\mathbb{R}^{n}$. Given an 
$n$-dimensional multi-index $\alpha$, the $\alpha$ th order partial derivative of $V$ is defined as $\partial^{[\alpha]} V=\frac{\partial^{\alpha_{1}} V}{\partial x_{1}} \frac{\partial^{\alpha_{2} V}}{\partial x_{2}} \ldots \frac{\partial^{\alpha_{n}}}{\partial x_{n}}$. The multi-index binomial theorem states

$$
(x+y)^{[\alpha]}=\sum_{0 \leq \beta \leq \alpha}\left(\begin{array}{c}
\alpha \\
\beta
\end{array}\right) x^{[\alpha-\beta]} y^{[\beta]}
$$

It can be shown that

$$
\partial^{[\alpha]} x^{[\beta]}= \begin{cases}\frac{\beta !}{(\beta-\alpha) !} x^{[\beta-\alpha]}, & \text { if } \alpha \leq \beta \\ 0 & \text { otherwise }\end{cases}
$$

A $p$ th order polynomial may be written in multi-index notation as $V(x)=\sum_{|\alpha| \leq p} c_{\alpha} x^{[\alpha]}$ where $\alpha$ is a multi-index and $c_{\alpha}$ is some coefficient associated with the $x^{[\alpha]}$ monomial. We say that the polynomial $V(x)$ is SOS or sum-of-squares if it can be rewritten as $V(x)=\sum_{k=1}^{M} q_{k}^{2}(x)$ of some set of $M$ polynomials $q_{k}(x)$ where $k=1,2, \ldots, M$.

\section{JUMP PROCESSES}

Consider a shot noise process

$$
J(t)=\sum_{\ell=1}^{N_{t}} y_{\ell} e^{-\delta\left(t-t_{\ell}\right)}
$$

where $\left\{y_{\ell}, \ell \in \mathbb{Z}_{+}\right\}$is an i.i.d. random process with distribution $F(y)$ describing the $\ell$-th jump's size, $\left\{\tau_{\ell}, \ell \in \mathbb{Z}_{+}\right\}$ are the event times of a Poisson process, $\left\{P(t), t \in \mathbb{R}_{+}\right\}$, with constant intensity $\rho$, and $\delta$ is a real positive constant representing a rate of exponential decay after a Poisson jump. Let $Y\left(\tau_{\ell}, y_{\ell}\right)=y_{\ell} e^{\delta \tau_{\ell}}$, then $J(t)$ may be written as

$$
J(t)=e^{-\delta t} \int_{0}^{t} \int_{\mathbb{R}^{n}} Y(\tau, y) N(d \tau, d y)
$$

where $N(d \tau, d y)$ is a Poisson random measure with $\mathbb{E}_{t}[N(d t, d y)]=\rho d t F(d y)$. We define the increment of the jump process $J$ as $d J(t)=J(t+d t)-J(t)$ where $d t$ is an infinitesimal time increment. Using equation 2 to expand out $d J(t)$ and retaining first order terms in $d t$, one finds the jump process increment can be written as

$$
d J(t)=-\delta J(t) d t+\int_{\mathbb{R}^{n}} y N(d t, d y)
$$

where the second term in equation (3) is known as a compound Poisson process.

Now consider a jump stochastic differential equation (JSDE)

$$
d x(t)=f(x(t)) d t+\sigma(x(t)) d w(t)+d J(t)
$$

where $f(\cdot): \mathbb{R}^{n} \rightarrow \mathbb{R}^{n}$ and $\sigma(\cdot): \mathbb{R}^{n} \rightarrow \mathbb{R}^{n}$ are Lipchitz continuous functions, $\left\{x(t), t \in \mathbb{R}_{+}\right\}$is a stochastic process, $\left\{w(t), t \in \mathbb{R}_{+}\right\}$is a Wiener process and $\left\{J(t), t \in \mathbb{R}_{+}\right\}$is the jump process defined in equation (2). Using the expression for the jump increment in equation (3), the JSDE can be rewritten as

$$
\begin{aligned}
d x(t)= & {[f(x(t))-\delta J(t)] d t+\sigma(x(t)) d w(t) } \\
& +\int_{\mathbb{R}^{n}} y N(d t, d y)
\end{aligned}
$$

One may observe that the solution of the JSDE consists of a diffusion process driven by a compound Poisson process. The diffusion process is Markov and the jump process in equation (1) is also Markov. Since $\{w(t)\}$ and $\{J(t)\}$ are independent of each other, one can conclude that the solution to the JSDE (5) will also be a Markov process [11].

Now consider a Markov process $\left\{x(t): t \in \mathbb{R}_{+}\right\}$and consider any function $V(\cdot, \cdot): \mathbb{R}_{+} \times \mathbb{R}^{n} \rightarrow \mathbb{R}$ that is twice differentiable in the second variable and differentiable in the first variable (denote this class of functions as $C^{1,2}$ ). Assume $\{x(t)\}$ has right continuous sample paths and consider the limit

$$
\lim _{h \downarrow 0} \frac{1}{h}\left[\mathbb{E}_{x} V(x(h))-V(x(0))\right]=\mathscr{L} V(x) .
$$

If this limit exists, we refer to it as the generator of the Markov process applied to the function $V$. When the process $x$ is a diffusion with continuous sample paths (i.e. $d x=$ $f(x) d t+\sigma(x) d w)$ then the generator, $\mathscr{L}$, is

$\mathscr{L} V(x)=\frac{\partial V(t, x)}{\partial x} f(x)+\frac{1}{2} \operatorname{Trace}\left(\sigma^{T}(x) \frac{\partial^{2} V(t, x)}{\partial x^{2}} \sigma(x)\right)$.

If the process $x$ is a jump process $d J$, then one can show [1] that the generator, $\mathscr{G}$, is

$$
\mathscr{G} V(x)=\rho \int_{0}^{\infty}(V(t, x+y)-V(t, x)) d F(y) .
$$

The following proposition characterizes the generator for the JSDE in equation (4).

Proposition 2.1: Let $V(\cdot, \cdot)$ be any $C^{1,2}$ real-valued function defined over $\mathbb{R}^{n}$. Assume that the jump process in equation (4) has right continuous sample paths, then the generator, $\mathscr{L}^{*}$, for this process is

$$
\begin{aligned}
\mathscr{L}^{*} V(t, x)= & \frac{\partial V(t, x)}{\partial x}[f(x(t))-\delta J(t)] \\
& +\frac{1}{2} \operatorname{Tr}\left(\sigma^{T}(x(t)) \frac{\partial^{2} V(t, x)}{\partial x^{2}} \sigma(x(t))\right) \\
& +\rho \int_{0}^{\infty}(V(t, x+y)-V(t, x)) d F(y)
\end{aligned}
$$

Proof: Since the generator is a linear operator, we can simply combine the results for the generators of the jump process and the diffusion process. $\diamond$.

\section{First PAssage Times}

Consider a random process, $\left\{x(t), t \in \mathbb{R}_{+}\right\}$, with $x(0)=x_{0}$. Consider an open subset $\mathscr{X}$ of $\mathbb{R}^{n}$ that contains $x_{0}$, and let $\partial \mathscr{X}$ denote the boundary of $\mathscr{X}$. The time at which a sample path of $x$ hits the boundary $\partial \mathscr{X}$ is a random variable $\tau$ called the first passage time. Formally, we can express this passage time as

$$
\tau \equiv \inf \{t \geq 0: x(t) \in \partial \mathscr{X}\}
$$

The standard approach for computing the expected value of $\tau$, i.e. mean first passage time (MFPT), is obtained by solving the corresponding backward Chapman-Kolmogorov equation [5]. This approach, however, is difficult to use for systems with dimensionality greater than one as it involves solving a set of partial differential equations with appropriate 
boundary conditions. An alternative approach for approximating the MFPT based on Itô's lemma is given by the following proposition.

Proposition 3.1: Consider a diffusion process $d x=$ $f(x) d t+\sigma d w$ defined on a bounded open subset $\mathscr{X} \subset \mathbb{R}^{n}$ with smooth boundary $\partial \mathscr{X}$. Assume the initial condition $x(0)$ takes its value in $\mathscr{X}_{0} \subset \mathbb{R}^{n}$. If there exists a real-valued function $V \in \mathscr{C}^{1,2}$ and a positive constant $\gamma>0$ such that

$$
\begin{aligned}
V(t, x) & \geq 0, \quad \forall x \in \mathscr{X} \\
V(t, x) & \leq 0, \quad \forall x \in \partial \mathscr{X} \\
V(t, x) & \leq \gamma, \quad \forall x \in \mathscr{X}_{0} \\
\frac{\partial V(t, x)}{\partial t}+\mathscr{L} V(t, x) & \leq-1, \quad \forall x \in \mathscr{X}
\end{aligned}
$$

where is the generator in equation (6), then $\mathbb{E}[\tau] \leq \gamma$ where $\tau=\inf \{t \geq 0: x(t) \in \partial \mathscr{X}\}$.

Proof: The technique for the proof is similar to that in [9]. Itô's lemma provides a stochastic differential equation for $V$

$$
d V(t, x)=\left(\frac{\partial V}{\partial t}+\mathscr{L} V\right) d t+\sum_{k=1}^{m}\left(\sum_{i=1}^{n} \frac{\partial V}{\partial x_{i}} \sigma_{i k}\right) d w_{k}(t)
$$

where $\mathscr{L} V$ is the generator of the diffusion $x$ with respect to function $V$. Let $\tau \equiv \inf \{t \geq 0: x(t) \in \partial \mathscr{X}\}$ and define $\tau \wedge t=$ $\min \{\tau, t\}$. Integrating $d V(t, x)$ from $[0, \tau \wedge t]$ and taking the expectation yields,

$$
\begin{aligned}
& \mathbb{E}[V(x(\tau \wedge t))] \\
& \quad=V(x(0))+\mathbb{E}\left[\int_{0}^{\tau \wedge t}\left(\frac{\partial V(s, x)}{\partial s}+\mathscr{L} V(s, x)\right) d s\right]
\end{aligned}
$$

Take the limit as $t \rightarrow \infty$ and using the last condition in the proposition's statement, one finds

$$
\mathbb{E}\left[V(x(\tau)] \leq V\left(x_{0}\right)-\mathbb{E}\left[\int_{0}^{\tau} d s\right]=V\left(x_{0}\right)-\mathbb{E}[\tau] .\right.
$$

$\tau$ is the first time the state trajectory hits the set $\partial \mathscr{X}$ and so equation (8) implies the MFPT satisfies

$$
\mathbb{E}[\tau] \leq V\left(x_{0}\right)-\mathbb{E}[V(x(\tau))]
$$

Boundary points of $\mathscr{X}$ are limit points of $\mathscr{X}$ and since $V(t, x) \geq 0$ on $\mathscr{X}$, this means $V(t, x)=0$ on $\partial \mathscr{X}$. One may therefore conclude that $\mathbb{E}[\tau, x(\tau)]=0$ which implies $\mathbb{E}[\tau] \leq$ $V(x(0))$. By the third condition, we know that $V(t, x) \leq \gamma$ on $\mathscr{X}_{0}$, which implies $\mathbb{E}[\tau] \leq \gamma . \diamond$

Remark 3.2: From the proof of this proposition, we see that $\mathbb{E}[V(x(t)) \mid V(x(0))] \leq V\left(x_{0}\right)$ for $0 \leq t \leq \tau$. Since $\mathscr{X}$ is bounded, this implies $\mathbb{E}[V(x(t))]<\infty$ which along with the requirement that $V(t, x) \geq 0$ implies the stochastic process generated by $V$ is a supermartingale.

The main result of this section is found in the following proposition, which uses the generator for the jump-diffusion process to approximate MFPT.

Proposition 3.3: Consider the jump diffusion process in equation (5) defined on a bounded open subset $\mathscr{X} \subset \mathbb{R}^{n}$ with smooth boundary $\partial \mathscr{X}$. Let the initial condition $x(0)$ is a random variable taking values in $\mathscr{X}_{0} \subset \mathbb{R}^{n}$. If there exists a real-valued function $V \in C^{1,2}$ and a positive constant $\gamma \geq 0$ such that

$$
\begin{aligned}
V(t, x) & \geq 0, \quad \forall x \in \mathscr{X} \\
V(t, x) & \leq 0, \quad \forall x \in \partial \mathscr{X} \\
V(t, x) & \leq \gamma, \quad \forall x \in \mathscr{X}_{0} \\
\frac{\partial V(t, x)}{\partial t}+\mathscr{L}^{*} V & \leq-1, \quad \forall x \in \mathscr{X}
\end{aligned}
$$

where $\mathscr{L}^{*}$ is the generator in equation (7), then $\mathbb{E}[\tau] \leq \gamma$ where $\tau=\inf \{t \geq 0: x(t) \in \partial \mathscr{X}\}$.

Proof: The proof is similar to that in Proposition (3.1) except that we use the generator described in Proposition (2.1). $\diamond$

Using the generator described in Proposition (2.1), one can also perform stochastic reachability analysis [9] for jumpdiffusion processes. Stochastic reachability aims to compute an upper bound for the probability that, starting in an initial set $\mathscr{X}_{0}$, the sample path of $\{x(t)\}$ will reach a given target set $\mathscr{X}_{T}$ in a finite time $t \leq \tau$, where $\tau=\inf \left\{t \geq 0: x(t) \in \mathscr{X}_{T}\right\}$. In other word, find a constant $\beta \in[0,1]$ such that

$$
\mathbb{P}\left[x(t) \in \mathscr{X}_{T} \text { for some } 0 \leq t \leq \tau \mid x(0) \in \mathscr{X}_{0}\right] \leq \beta .
$$

This problem was solved for diffusion processes in [9]. The solution involves searching for a "stochastic" Lyapunov function, $V(t, x)$, that generates a supermartingale from which an upper bound on $\beta$ can be deduced. Using the process generator in (7), the same method from [9] can be used to extend this search for jump diffusion processes (5). This extension is stated in the following proposition.

Proposition 3.4: Consider the jump-diffusion process in equation (5). Let $T$ be a finite time horizon and let $\mathscr{X}, \mathscr{X}_{0}$, and $\mathscr{X}_{T}$ be bounded subsets of $\mathbb{R}^{n}$. If there exists a function $V(t, x) \in \mathscr{C}^{1,2}$ and a constant $\beta \in[0,1]$ such that

$$
\begin{aligned}
V(t, x) & \leq \beta, \quad \forall(x, t) \in \mathscr{X}_{0} \times[0, T] \\
V(t, x) & \geq 1, \quad \forall(x, t) \in \mathscr{X}_{T} \times[0, T] \\
V(t, x) & \geq 0, \quad \forall(x, t) \in \mathscr{X} \times[0, T] \\
\frac{\partial V(t, x)}{\partial t}+\mathscr{L}^{*} V(t, x) & \leq 0, \quad \forall(x, t) \in \mathscr{X} \times[0, T]
\end{aligned}
$$

where $\mathscr{L}^{*} V(t, x)$ is defined as in Proposition 2.1, then

$$
\mathbb{P}\left[x(t) \in \mathscr{X}_{T} \text { for some } 0 \leq t \leq \tau \mid x(0) \in \mathscr{X}_{0}\right] \leq \beta .
$$

Proof: The proof is similar to that in [9, Th. 15] except that we use the generator described in Proposition 2.1. $\diamond$

\section{SOS PROGRAMMING}

The propositions in section III show that characterizing a jump process' first passage times involves finding a function $V(t, x)$ that is positive/negative semi-definite on appropriately defined subsets of $\mathbb{R}^{n}$. Searching for functions that are positive definite, however, can be difficult. Since any SOS polynomial is also positive semi-definite, one can relax the positive definite conditions in proposition 3.4 to simply requiring that the functions be SOS. Once this is done, then one can establish the positive-definiteness of the functions by using semi-definite programming to search for 
SOS functions [8]. In particular, if the vector fields in (5) are polynomials and the sets $\left\{\mathscr{X}, \mathscr{X}_{0}, \partial \mathscr{X}, \mathscr{X}_{T}\right\}$ can be described as polynomial equalities/inequalities, the search for $V(t, x)$ can be cast as SOS program which can be solved efficiently using SOSTOOLS [10] combined with a semidefinite program solver [15].

An SOS program is a convex optimization problem of the form

$$
\begin{array}{ll}
\min & \sum_{j=1}^{m} \omega_{j} c_{j} \\
\text { s.t. } & p(x)=\sum_{\alpha \leq p} c_{\alpha} x^{[\alpha]} \text { is SOS, for } \alpha=\{0, \ldots, p\}
\end{array}
$$

where $\omega_{j}$ 's are given real numbers, $c_{j}$ 's are scalar real decision variables, $x^{[\alpha]}$ is a given $[\alpha]$-th degree polynomial in $n$-variables, and $\alpha$ is an $n$-dimensional multi-index.

To search for $V(t, x)$ that satisfies each condition in Propositions $3.1,3.3$ and 3.4 using SOS programming, we will first need to express each inequality in $V(t, x)$ as a positive definiteness condition for $V(t, x)$ and then replace them with SOS condition. Replacement from positive definite condition to SOS condition can be done by introducing other SOS multipliers whose coefficients will also be determined during the optimization task. The following proposition describes the SOS program for Proposition 3.1

Proposition 4.1: Consider the diffusion process $d x=$ $f(x) d t+\sigma d w$ with initial $x(0)=x_{0}$. Let the sets $\mathscr{X}, \mathscr{X}_{0}$, $\partial \mathscr{X}$ be described by $\mathscr{X}=\left\{x \in \mathbb{R}^{n}: g_{\mathscr{X}}(x) \geq 0\right\}, \mathscr{X}_{0}=\{x \in$ $\left.\mathbb{R}^{n}: g_{\mathscr{X}_{0}}(x) \geq 0\right\}, \partial \mathscr{X}=\left\{x \in \mathbb{R}^{n}: g_{\partial \mathscr{X}}(x)=0\right\}$, respectively, where $g$ 's are polynomials. Consider a polynomial parameterization $\mathscr{V}$ of $V(t, x)$, and define $\tau \equiv \inf \{t \geq 0: x(t) \in \partial \mathscr{X}\}$. If there exists $V(t, x) \in \mathscr{V}$, a constant $\gamma>0$, a small constant $\varepsilon>0$, and SOS polynomials $\sigma_{\mathscr{X}}(x), \sigma_{\mathscr{X}_{0}}(x), \sigma_{\partial \mathscr{X}}(x)$ such that the following SOS program

$$
\begin{array}{ccc}
\min & \gamma & \\
\text { s.t. } & V(t, x)-\sigma_{\mathscr{X}}(x) g_{\mathscr{X}}(x)-\varepsilon & \text { is SOS, } \\
& -V(t, x)-\sigma_{\partial \mathscr{X}}(x) g_{\partial \mathscr{X}}(x) & \text { is SOS, } \\
& -V(t, x)+\gamma-\sigma_{\mathscr{X}_{0}}(x) g_{\mathscr{X}_{0}}(x) & \text { is SOS, } \\
& -\frac{\partial V(t, x)}{\partial t}-\mathscr{L} V(t, x)-\sigma_{\mathscr{X}}(x) g_{\mathscr{X}}(x)-1 & \text { is SOS, }
\end{array}
$$

has a feasible solution, then $E[\tau] \leq \gamma$.

Proof: The conditions for $V(t, x)$ in the above SOS program is the SOS relaxation of the inequalities in Proposition 3.1. Such relaxation for each corresponding set is accommodated using the SOS multipliers $\sigma_{(\cdot)}(x)$. Also, $\gamma(0-$ order SOS polynomial) is chosen as the objective function to be minimized since its minimum value obtained from the optimization will serve as the tightest upper bound for a given SOS polynomial parameterization. $\diamond$

The inequality constraints in proposition 3.3 cannot be directly translated into SOS constraints due to the presence of integral term in the generator. The following proposition shows how to rewrite the inequality constraint on generator $\mathscr{L}^{*} V$ as an SOS polynomial.

Proposition 4.2: Let $y \in \mathbb{R}^{n}$ be an $n$-dimensional independent random variable with distribution $F(y)$. Let $V(x)=$ $\sum_{|\alpha| \leq p} c_{\alpha} x^{[\alpha]}$ be a multi-index representation of a polynomial function. Then

$$
\int(V(x+y)-V(x)) d F(y)=\sum_{1 \leq|\beta| \leq p} \frac{1}{\beta !} \partial^{[\beta]}[V(x)] \mathbb{M}^{|\beta|}
$$

\section{Proof:}

$$
\begin{aligned}
V(x+y) & =\sum_{|\alpha| \leq p} c_{\alpha}(x+y)^{[\alpha]} \\
& =\sum_{|\alpha| \leq p} c_{\alpha} \sum_{0 \leq|\beta|, \beta \leq \alpha}\left(\begin{array}{c}
\alpha \\
\beta
\end{array}\right) x^{[\alpha-\beta]} y^{[\beta]} \\
& =\sum_{|\alpha| \leq p} c_{\alpha}\left[x^{[\alpha]}+\sum_{1 \leq|\beta|, \beta \leq \alpha}\left(\begin{array}{c}
\alpha \\
\beta
\end{array}\right) x^{[\alpha-\beta]} y^{[\beta]}\right]
\end{aligned}
$$

For notational convenience, let us denote the difference $V(x+y)-V(x)$ as $\Delta V(x, y)$. Using the above sum, one can write this difference as

$$
\Delta V(x, y)=\sum_{|\alpha| \leq p} c_{\alpha} \sum_{1 \leq|\beta|, \beta \leq \alpha}\left(\begin{array}{l}
\alpha \\
\beta
\end{array}\right) x^{[\alpha-\beta]} y^{[\beta]}
$$

and since

$$
\partial^{[\beta]}\left[x^{[\alpha]}\right]=\left\{\begin{array}{cl}
\frac{\alpha !}{(\alpha-\beta) !} x^{[\alpha-\beta]} & \text { if } \beta \leq \alpha \\
0 & \text { otherwise }
\end{array}\right.
$$

the expression for $\Delta V$ can be rewritten as

$$
\Delta V(x, y)=\sum_{|\alpha| \leq p} c_{\alpha} \sum_{1 \leq|\beta|, \beta \leq \alpha} \frac{1}{\beta !} \partial^{[\beta]}\left[x^{[\alpha]}\right] y^{[\beta]}
$$

Expand out the first summation to obtain

$$
\begin{aligned}
\Delta V(x, y)= & \sum_{|\alpha|=1} c_{\alpha} \sum_{|\beta|=1} \frac{1}{\beta !} \partial^{[\beta]}\left[x^{[\alpha]}\right] y^{[\beta]} \\
& +\sum_{|\alpha|=2} c_{\alpha} \sum_{1 \leq|\beta| \leq 2} \frac{1}{\beta !} \partial^{[\beta]}\left[x^{[\alpha]}\right] y^{[\beta]} \\
& \vdots \\
& +\sum_{|\alpha|=p} c_{\alpha} \sum_{1 \leq|\beta| \leq p} \frac{1}{\beta !} \partial^{[\beta]}\left[x^{[\alpha]}\right] y^{[\beta]}
\end{aligned}
$$

The order of the summations can now be interchanged since $\alpha$ and $\beta$ are no longer directly coupled to yield

$$
\begin{aligned}
\Delta V(x, y)= & \sum_{|\beta|=1} \frac{1}{\beta !}\left[\sum_{|\alpha|=1} c_{\alpha} \partial^{[\beta]}\left[x^{[\alpha]}\right]\right] y^{[\beta]} \\
& +\sum_{1 \leq|\beta| \leq 2} \frac{1}{\beta !}\left[\sum_{|\alpha|=2} c_{\alpha} \partial^{[\beta]}\left[x^{[\alpha]}\right]\right] y^{[\beta]} \\
& \vdots \\
& +\sum_{1 \leq|\beta| \leq p} \frac{1}{\beta !}\left[\sum_{|\alpha|=p} c_{\alpha} \partial^{[\beta]}\left[x^{[\alpha]}\right]\right] y^{[\beta]}
\end{aligned}
$$


Reordering the terms in the first summation yields,

$$
\begin{aligned}
\Delta V(x, y)= & \sum_{|\beta|=1} \frac{1}{\beta !}\left[\sum_{1 \leq|\alpha| \leq p} c_{\alpha} \partial^{[\beta]}\left[x^{[\alpha]}\right]\right] y^{[\beta]} \\
& +\sum_{|\beta|=2} \frac{1}{\beta !}\left[\sum_{2 \leq|\alpha| \leq p} c_{\alpha} \partial^{[\beta]}\left[x^{[\alpha]}\right]\right] y^{[\beta]} \\
& \vdots \\
& +\sum_{|\beta|=p} \frac{1}{\beta !}\left[\sum_{|\alpha|=p} c_{\alpha} \partial^{[\beta]}\left[x^{[\alpha]}\right]\right] y^{[\beta]}
\end{aligned}
$$

Because $\partial^{[\beta]}\left[x^{[\alpha]}\right]=0$ when $\alpha \leq \beta$, the summation limits of the inner sums can be extended from 1 to $p$ thereby yielding

$$
\begin{aligned}
\Delta V(x, y)= & \sum_{|\beta|=1} \frac{1}{\beta !}\left[\sum_{1 \leq|\alpha| \leq p} c_{\alpha} \partial^{[\beta]}\left[x^{[\alpha]]}\right] y^{[\beta]}\right. \\
& +\sum_{|\beta|=2} \frac{1}{\beta !}\left[\sum_{1 \leq|\alpha| \leq p} c_{\alpha} \partial^{[\beta]}\left[x^{[\alpha]}\right]\right] y^{[\beta]} \\
& \vdots \\
& +\sum_{|\beta|=p} \frac{1}{\beta !}\left[\sum_{1 \leq|\alpha| \leq p} c_{\alpha} \partial^{[\beta]}\left[x^{[\alpha]]}\right] y^{[\beta]}(10)\right.
\end{aligned}
$$

Now note that

$$
\partial^{[\beta]} V(x)=\partial^{[\beta]}\left[\sum_{|\alpha| \leq p} c_{\alpha} x^{[\alpha]}\right]=\sum_{1 \leq|\alpha| \leq p} c_{\alpha} \partial^{[\beta]}\left[x^{[\alpha]}\right]
$$

which is simply the inner sum in (10) and so the difference becomes

$$
\Delta V(x, y)=\sum_{1 \leq|\beta| \leq p} \frac{1}{\beta !} \partial^{[\beta]}[V(x)] y^{[\beta]}
$$

Integrating both sides with respect to $F(y)$, and since each component of $y$ is independent, we have

$$
\begin{aligned}
\int \Delta V(x, y) d F(y) & =\sum_{1 \leq|\beta| \leq p} \frac{1}{\beta !} \partial^{[\beta]}[V(x)] \int y^{|\beta|} d F(y) \\
& =\sum_{1 \leq|\beta| \leq p} \frac{1}{\beta !} \partial^{[\beta]}[V(x)] \mathbb{M}|| \beta \mid
\end{aligned}
$$

$\diamond$

Using Proposition (4.2), the JSDE's generator in Proposition (2.1) can be rewritten as

$$
\begin{aligned}
\mathscr{L}^{*} V(t, x)= & \frac{\partial V(t, x)}{\partial x}[f(x(t))-\delta J(t)] \\
& +\frac{1}{2} \operatorname{Tr}\left(\sigma^{T}(x(t)) \frac{\partial^{2} V(t, x)}{\partial x^{2}} \sigma(x(t))\right) \\
& +\rho \sum_{1 \leq|\beta| \leq p}^{p} \frac{1}{\beta !} \partial^{[\beta]}[V(x)] \mathbb{M}^{|\beta|}
\end{aligned}
$$

With this generator, propositions 3.3 and 3.4 can be used to bound the MFPT and passage time probability using SOS programs that only require knowledge of the partial derivatives of the candidate function $V$.

\section{ECO-System MANAGEMENT}

First passage times (FPT) may be used as a measure of an eco-system's resilience. This section uses the FPT approximations discussed above to manage eco-systems. In particular, we consider the problem of choosing a harvesting strategy to manage the bass-crayfish population in freshwater lakes.

Bass-crayfish interaction is an intraguild-predation (IGP) type in which both species compete for the same resource while at the same time the crayfish predate the bass. This system has two equilibria; one in which the bass dominate the eco-system and the other in which the crayfish dominate the eco-system. An outbreak of crayfish is undesirable as it can suppress the bass population. If such an outbreak occurs, management strategies are needed to induce a regime shift back to the bass-dominated equilibrium point.

One method for achieving this management objective is to permit the harvesting of crayfish by anglers. In general, this harvesting process can be modeled as a compound jump process in which the size of harvest and the intensity of harvesting events are the variables that the eco-system manager needs to set. The FPT analysis presented in the preceding sections provides a systematic framework in which such management decisions can be made.

This particular example is drawn from a paper [2] that used mean first passage times as a basis for management decisions. The underlying system model is characterized by the following state equations,

$$
\begin{aligned}
& \dot{x}_{1}(t)=x_{1}\left(1-x_{1}-0.7 x_{2}\right)-\frac{0.075 x_{2} x_{1}^{2}}{0.01+x_{1}^{2}}-\sum_{i=1}^{N_{t}} y_{i} \delta\left(t-\tau_{i}\right) \\
& \dot{x}_{2}(t)=1.5 x_{2}\left(1-0.9 x_{1}-x_{2}\right)+0.01 \frac{0.075 x_{2} x_{1}^{2}}{0.01+x_{1}^{2}}
\end{aligned}
$$

in which the biomass of the crayfish and bass are denoted as $x_{1}$ and $x_{2}$, respectively. The last term in the first equation models crayfish harvesting as a double exponential jump process in which $N_{t}$ is the number of harvest events in the interval $[0, t]$, the harvest size $\left\{y_{i}\right\}_{i=1}^{N_{t}}$ and the harvest times, $\left\{\tau_{i}\right\}_{i=1}^{N_{t}}$ are exponentially distributed i.i.d. random processes with intensity $\mu$, and $\lambda$, respectively.

In the absence of harvesting, this model has three equilibria (two stable and one unstable) in $\mathbb{R}_{+}^{2}$. One of these equilibria is dominated by the bass and the other is dominated by the crayfish. The stable equilibria have regions of attraction (ROA) that partition the state space into two regions as shown in Figure 1. This figure plots the isoclines for equation (12), identifies the two stable equilibrium, and marks the separatrix between the two competing regions of attraction.

Figure 1 also shows sample path for this system under a given harvesting policy. From this figure, one sees that each harvesting event causes a step decrease in the crayfish population, after which the system begins relaxing back to the crayfish-dominated equilibrium. There is a finite probability that repeated harvesting events will drive the system state across the separatrix, whereupon the system's equilibrium 
state shifts to the bass-dominated equilibrium. Assuming that the system's current state lies in the ROA dominated by the crayfish, we're interested in specifying those harvesting parameters $\mu$ and $\lambda$ that maximize the probability of a regime shift to the bass-dominated ROA in a specified timeinterval subject to a constraint that limits the probability of driving the crayfish population to extinction.

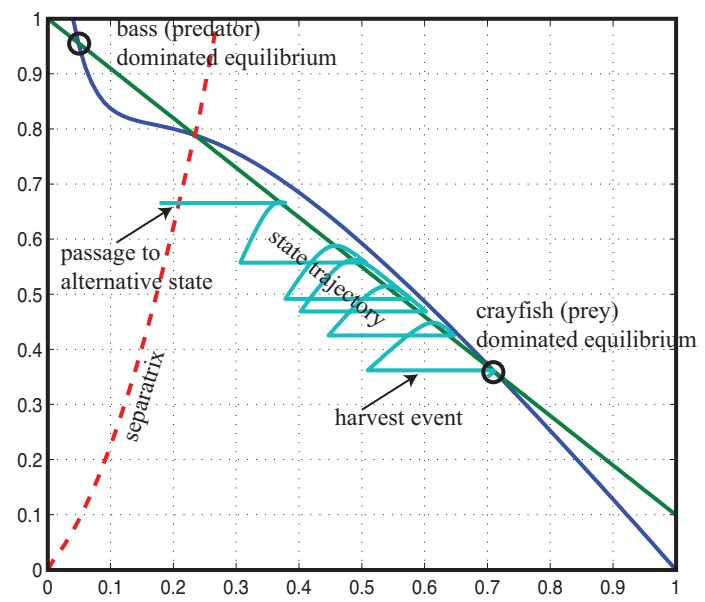

Fig. 1: Regions of Attraction in Bass-Crayfish Eco-system [2]

Approximations to the MFPT are obtained with the SOS program in proposition $4.1 \mathrm{using}$ the generator representation developed in proposition 4.2. In particular, we define the following sets,

$$
\left.\begin{array}{rl}
\mathscr{X} & =\left\{x \in \mathbb{R}_{+}^{2}, t \in \mathbb{R}_{+} \mid \begin{array}{l}
x_{1}\left(1-x_{1}\right) \geq 0, \quad x_{2}(1- \\
\left.x_{2}\right) \geq 0, t(T-t)>0
\end{array}\right\} \\
\mathscr{X}_{0} & =\left\{x \in \mathbb{R}_{+}^{2}, \mid \begin{array}{l}
-(x-0.72)^{2}-\left(x_{2}-\right. \\
0.36)^{2}+(0.01)^{2} \geq 0
\end{array}\right\} \\
\partial \mathscr{X} & =\left\{x \in \mathbb{R}_{+} \mid \begin{array}{l}
x_{1}\left(0.27-x_{1}\right) \geq 0, x_{2}(1- \\
\left.x_{2}\right) \geq 0, x_{2}-0.14 x_{1}^{3}- \\
9.5 x_{1}^{2}-1.1 x_{1}+0.0003=0
\end{array}\right.
\end{array}\right\}
$$

Region $\mathscr{X}$ characterizes a unit square in $\mathbb{R}_{+}^{2}$ over the time interval $[0, T]$. The initial region, $\mathscr{X}_{0}$ is a disk centered at the crayfish-dominated equilibrium with a radius of 0.01 . The boundary region, $\partial \mathscr{X}$ is the separatrix shown in Figure 1.

The SOSTOOLS were used to find an SOS polynomial, $V(t, x)$, that satisfies the SOS program in proposition 4.1 for a range of $\mu$ and $\lambda$. Figure 2 shows the MFPT approximation (circle) for $\mu=0.1$ and $\lambda$ between 0 to 6 . This plot also shows the MFPT obtained using a Monte Carlo simulation with a 95\% confidence interval and estimates (asterisk) of the MFPT obtained in [2]. The MFPT estimates obtained in [2] were based on a linearization and were only valid for small mean harvest sizes $(\mu)$. As a result, the estimates from [2] under approximate the actual MFPT seen in Monte Carlo simulations, whereas our results provide reasonable upper bounds on the MFPT.

A more complete surface plot showing our MFPT approximations for a range of $\mu$ and $\lambda$ is shown in Figure 3. This

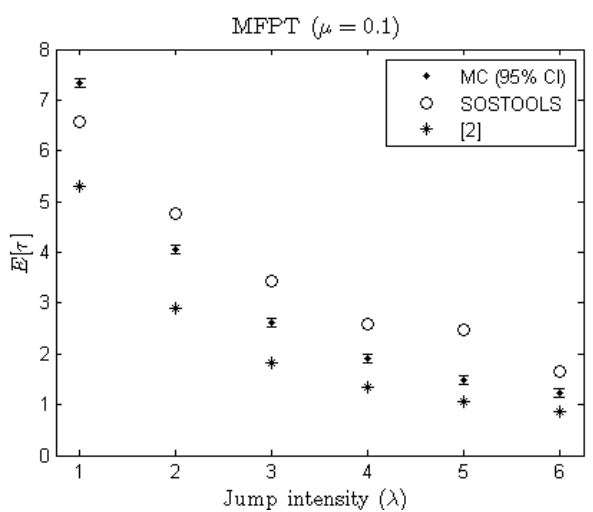

Fig. 2: MFPT comparison for $\mu=0.1$ and various $\lambda$.

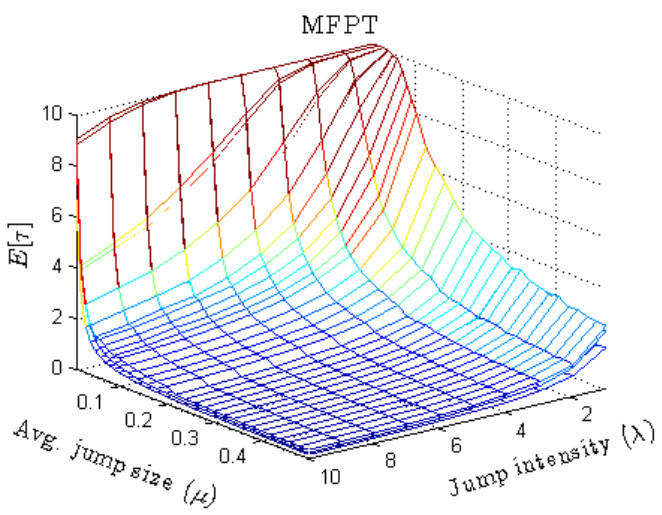

Fig. 3: MFPT as a function of $\mu$ and $\lambda$

plot may be used as the basis for determining a harvesting policy provided some additional constraints are imposed. Clearly, one can maximize the likelihood of a regime shift by simply increasing the intensity of harvesting. In general, however, one would want to limit such harvesting intensity. One issue with large harvesting intensity is that it may drive the crayfish population to extinction. While crayfish may be considered to be a "nuisance", the extinction of a species in the eco-system reduces overall bio-diversity and often makes such systems more prone to collapse from extreme events [7]. A reasonable limit on harvesting intensity involves limiting the harvesting rates $\lambda$ and $\mu$ to minimize the likelihood of crayfish extinction while still achieving a regime shift over a specified time interval.

Computation of extinction probabilities and regime shift probabilities can also be done using the SOS programming methods introduced in the preceding section. For initial states around the crayfish dominated equilibrium, we compute two probabilities. The first one is the probability of reaching a controlled region (i.e. $\partial \mathscr{X}$ ) which we denote as $\mathbb{P}_{R}$. The second one is the probability of reaching a small neighborhood of $x_{1}=0$, which corresponds to the event that the crayfish will be driven to extinction. This extinction probability is denoted as $\mathbb{P}_{E}$. We now define an SOS program in which the initial set, $\mathscr{X}_{0}$, and domain $\mathscr{X}$ are the same as before. To compute an upper bound $\beta_{R}$ for the regime shift probability, 


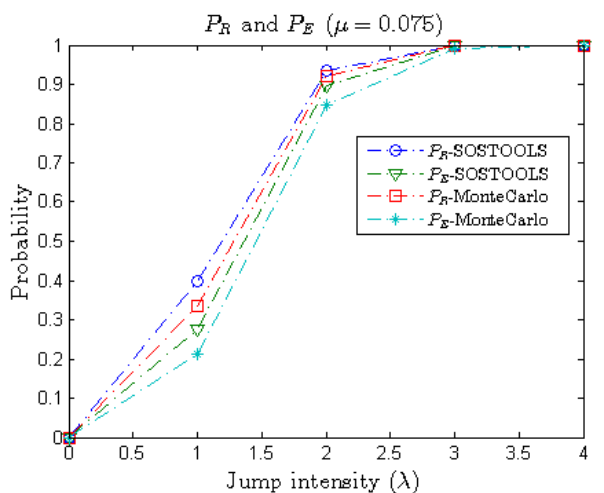

Fig. 4: $\mathbb{P}_{R}$ and $\mathbb{P}_{E}$ for $\mu=0.075$.

$\mathbb{P}_{R}$, we define the target set

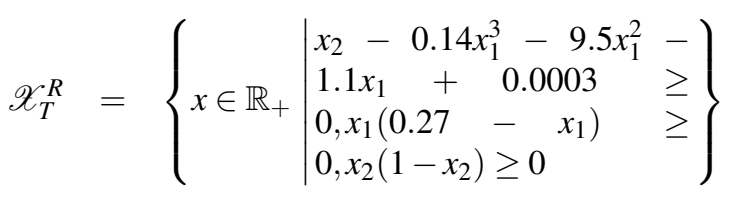

whereas for an upper bound $\beta_{E}$ on the extinction probability, $\mathbb{P}_{E}$, the target set is

$$
\mathscr{X}_{T}^{E}=\left\{\begin{array}{l|l}
x \in \mathbb{R}_{+}^{2} & \begin{array}{l}
x_{1}\left(0.01-x_{1}\right) \geq 0, x_{2}(1- \\
\left.x_{2}\right) \geq 0
\end{array}
\end{array}\right\}
$$

A similar computation is then done using the SOSTOOLS to obtain upper bounds $\beta_{R}$ and $\beta_{E}$ on the regime shift and extinction probabilities. A plot of this result is shown in Figure 4 for a specific harvest size $\mu=0.075$ and a range of $\lambda$ for a specified time interval of $T=5$.

The preceding approximations can be used in many ways. One possible management strategy would be to determine those intensities $\mu$ and $\lambda$ that minimize the probability of crayfish extinction subject to a constraint requiring the regime shift's MFPT to be less than a specified deadline, $T$. Let us set, for example, $T=5$ as the deadline for regime shift's MFPT and search for that $(\mu, \lambda)$ that give the smallest upper bound, $\beta_{E}$, of the extinction probability $\mathbb{P}_{E}$. Using the surface plot in Fig. 2, we first find all possible $(\mu, \lambda)$ pairs for which $\mathbb{E}[\tau] \leq 5$. Among all of these sets, we then choose one that gives the smallest $\beta_{E}$. We find that the pair $(\mu=0.075, \lambda=1)$ gives $\mathbb{E}[\tau] \leq 4.31$ and $\mathbb{P}_{E} \leq \beta_{E}=0.277$ (triangle in Figure 4). Using this pair, we use a Monte Carlo simulation with $95 \%$ confidence interval to compute the corresponding MFPT and $\mathbb{P}_{E}$. We find that MFPT $=$ $4.6896 \pm 0.083$ and $\mathbb{P}_{E}=0.243 \pm 0.104$. This confirms that our management approach minimizes the likelihood of an extinction event while assuring the regime shift's MFPT is less than the specified deadline of $T=5$.

One may also compare our proposed management framework with the method used in [2]. The approach in [2] uses an analytical formula to compute the MFPT for a linearization of (12). Since [2] does not provide a way to compute $\mathbb{P}_{E}$ for a given $(\mu, \lambda)$, we ran a Monte Carlo simulation to compute $\mathbb{P}_{E}$ for all these possible parameters and then singled out a parameter pair giving the smallest $\mathbb{P}_{E}$. The minimizing pair was $(\mu=0.055, \lambda=1)$ with an extinction probability of $\mathbb{P}_{E}=0.174$ and a MFPT of $6.4008 \pm 0.083$, which clearly violates the specified MFPT deadline.

\section{CONCLUding REMARKS}

This paper presented a computational method to characterize eco-system regime shifts under stochastic environmental forces. SOS programming was used to characterize the first passage times of a jump diffusion process. When used as a basis for managing regime shifts in eco-systems, our approach performs better than prior work that relies on model linearization. The models used in this work assume a spatially homogeneous eco-system. Future work will extend the proposed framework to spatially heterogeneous ecosystems.

\section{REFERENCES}

[1] R. Cont and P. Tankov. Financial modelling with jump processes, volume 2. Chapman \& Hall/CRC, 2003.

[2] K. L. S. Drury and D. M. Lodge. Using mean first passage times to quantify equilibrium resilience in perturbed intraguild predation systems. Theoretical Ecology, 2(1):41-51, 2009.

[3] H. El-Samad, S. Prajna, A. Papachristodoulou, J. Doyle, and M. Khammash. Advanced methods and algorithms for biological networks analysis. Proceedings of the IEEE, 94(4):832-853, 2006.

[4] C. Folke, S. Carpenter, B. Walker, M. Scheffer, T. Elmqvist, L. Gunderson, and CS Holling. Regime shifts, resilience, and biodiversity in ecosystem management. Annual Review of Ecology, Evolution, and Systematics, pages 557-581, 2004.

[5] C. W. Gardiner. Handbook of Stochastic Methods: For Physics, Chemistry, and the Natural Sciences. Springer-Series, New York, 1985.

[6] C.S. Holling. Resilience and stability of ecological systems. Annual review of ecology and systematics, pages 1-23, 1973.

[7] C.S. Holling. Engineering versus ecological resilience. In P.C. Schulze, editor, Engineering with ecological constraints, pages 3144. National Academy Press, Washington D.C., 1996.

[8] P.A. Parrilo. Structured semidefinite programs and semialgebraic geometry methods in robustness and optimization. $\mathrm{PhD}$ thesis, California Institute of Technology, Pasadena, CA, 2000.

[9] S. Prajna, A. Jadbabaie, and G.J. Pappas. A framework for worst-case and stochastic safety verification using barrier certificates. Automatic Control, IEEE Transactions on, 52(8):1415-1428, 2007.

[10] S. Prajna, A. Papachristodoulou, P. Seiler, and P. A. Parrilo. SOSTOOLS: Sum of squares optimization toolbox for MATLAB, 2004.

[11] P. Protter. Stochastic Integration and Differential Equations: Version 2.1, volume 21. Springer, 2005.

[12] Ann K Sakai, Fred W Allendorf, Jodie S Holt, David M Lodge, Jane Molofsky, Kimberly A With, Syndallas Baughman, Robert J Cabin, Joel E Cohen, Norman C Ellstrand, et al. The population biology of invasive specie. Annual Review of Ecology and Systematics, pages 305-332, 2001.

[13] M. Scheffer, S. Carpenter, J.A. Foley, C. Folke, B. Walker, et al. Catastrophic shifts in ecosystems. Nature, 413(6856):591-596, 2001.

[14] M. Scheffer, SH Hosper, ML Meijer, B. Moss, and E. Jeppesen. Alternative equilibria in shallow lakes. Trends in Ecology \& Evolution, 8(8):275-279, 1993.

[15] J.F. Sturm. Using sedumi 1.02, a matlab toolbox for optimization over symmetric cones. Optimization methods and software, 11(1-4):625653, 1999. 